\title{
Extensive Tissue Necrosis: A Rare but Catastrophic Complication of Sclerotherapy
}

\author{
Paul Robert Weaver* \\ President of the New Zealand Society of Procedural Phlebology, New Zealand
}

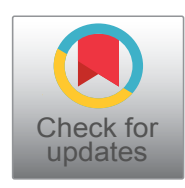

*Corresponding author: Paul Robert Weaver, MBBS (Lond.) Dip. Obst. (Otago) F.R.N.Z.C.G.P, President of the New Zealand Society of Procedural Phlebology, Medical Director, 0800 VEIN DR, 811 Heretaunga St. East, Parkvale, Hastings, Hawke's Bay, 4122, New Zealand, Tel: +64-274538931, Fax: +64-8710829

\section{Introduction}

Both sclerotherapy and open surgical treatment have been used for varicose veins for over 100 years [1]. Increasingly in the last 20 years the non-surgical treatments, such as endovenous ablation (EVA) and sclerotherapy, have became popular and largely replaced open surgical treatments in international guidelines [2]. In my practice, sclerotherapy has developed from a cosmetic treatment for superficial veins, into a commonly used therapy for saphenous trunks and tributaries [1]. Ultrasound guided foam sclerotherapy (UGFS) has become an established modality for the treatment of varicose veins, either alone or in combination with other non-surgical treatments.

While sclerotherapy may seem safe, catastrophic complications have been reported, including local and systemic adverse reactions. For example, extensive tissue necrosis and cerebrovascular accident (stroke) [3].

Patient selection is vital and treating physicians should be aware of all potential complications and discuss them with their patients, prior to treatment, to obtain informed consent. Physicians also need to be prepared to manage catastrophic complications that could lead to amputation or death.

\section{Tissue Necrosis}

Intra-arterial injection represents the most feared complication of sclerotherapy for varicose veins [4].

Ulceration or local tissue necrosis is rare following sclerotherapy and in the region of 0.2 to $1.2 \%$ [5]. The aetiology is poorly understood and thought to be due to extravasation of sclerosant during injection. It is my experience that sclerotherapy ulcers heal by secondary intention and good wound management to encourage granulation. They are usually quite painful and warrant simple analgesia and reassurance.

Extensive tissue necrosis has been ascribed to direct intra-arterial injection and is extremely rare with $<100$ cases reported worldwide. However, despite several different treatment approaches, amputation could not be prevented in $>50 \%$ of cases. Inadvertent intra-arterial injection represents a limb threatening complication of sclerotherapy [4]. Ischaemia of 6 hours duration [6] will lead to extensive tissue necrosis, there by necessitating early recognition and treatment to promote revascularisation.

The signs and symptoms of acute limb ischaemia include the six p's. These are pain, pallor, pulselessness, perishing cold, paraesthesia and paralysis [6]. Painful injection associated with immediate pallor of the affected limb should raise the suspicion of a possible inadvertent arterial injection. Over the following hours the pallor turns to a demarcated, mottled area associated with the affected peripheral artery. However, this is variable and sometimes there is no preceding pain or pallor.

The evidence for direct intra-arterial injection is circumstantial and there are other theories to explain the flow of sclerosant into the arterial arborisation. Arteriovenous malformations and thermoregulatory

\footnotetext{
Citation: Weaver PR (2020) Extensive Tissue Necrosis: A Rare but Catastrophic Complication of Sclerotherapy. Int Arch Vasc Med 3:005. doi.org/10.23937/2690-3164/1710005

Accepted: March 10, 2020: Published: March 12, 2020

Copyright: (C) 2020 Weaver PR. This is an open-access article distributed under the terms of the Creative Commons Attribution License, which permits unrestricted use, distribution, and reproduction in any medium, provided the original author and source are credited.
} 
shunts (damaged by venous hypertension) have been postulated [7]. Equally the Venoarteriolar Reflex, where arteries constrict in response to rapid dilatation of their corresponding veins, has been put forward as an explanation of how arterial ischaemia can occur despite obvious intravenous injection of sclerosant on duplex ultrasound [8]. This effect has also been shown to occur in the contralateral limb [9] and it is not hard to imagine that if the patient's microcirculation is already compromised [10] this could lead to devastating consequences. Never more so than a recent patient of mine in her 70's, with strong cardiovascular risk factors, who developed bilateral ischaemic feet and suffered bilateral below knee amputation. I had treated her with bilateral UGFS as an adjunct to bilateral radio-frequency thermal ablation (RFA.)

\section{So how can these potentially devastating complications be avoided?}

The first area of concern is patient selection. There is varying opinion around the world regarding peripheral arterial disease (PAD) as a contra-indication for sclerotherapy. However professional bodies in India in 2011 and the USA in 2014 determined severe PAD to be an absolute contra-indication to sclerotherapy treatment [2]. My own patient had several cardiovascular risk factors such as age (> $70 \mathrm{yrs}$ ), smoking, hypertension, hypercholesterolaemia and strong family history of ischaemic heart disease but was asymptomatic for PAD. With years of experience and success, treating all comers for varicose veins, it is possible for phlebologists to overlook the risks involved with treating arteriopaths and patients with other chronic diseases. I now believe that asymptomatic patients with risk factors and symptomatic PAD patients should be further investigated prior to sclerotherapy. Investigations to be considered, in the office setting, are evaluation of the peripheral pulses [11] and duplex scanning of Doppler waveforms and pulse volume recordings [12,13]. A normal lower extremity arterial Doppler velocity tracing is triphasic. A biphasic signal is considered abnormal if there is a clear transition from triphasic to biphasic along the vascular tree and monophasic waveforms are always considered abnormal [12]. The ankle-brachial (ABI) and toe-brachial (TBI) indices are also easily performed and have high sensitivity and specificity for PAD.

The next area to consider is the sclerotherapy treatment itself. Unfortunately, the data associated with sclerotherapy treatment guidelines is heterogeneous and there is huge variation in technique between practitioners and in different countries. I believe that one important area to consider is the maximum volume of sclerosant per session. As regards foam volume, $10 \mathrm{ml}$ is the upper limit for European guidelines and $20 \mathrm{ml}$ for the Australian guidelines [2]. It would seem prudent to use the minimum possible dose, volume and concentration required per session. Consideration should be given to treating unilaterally rather than bilaterally as a measure to reduce potential complications and keeping the volume of foam to $<10 \mathrm{ml}$ per session.

When it comes to treating saphenous, axial incompetence and especially in large veins $(>10 \mathrm{~mm}$ diameter at the saphenofemoral or saphenopopliteal junctions) I am increasingly combining thermal and non-thermal EVA techniques to reduce the reliance on UGFS. For example, in a large great saphenous vein I will combine RFA proximally with cyanoacrylate glue ablation distally and delay any further treatment for a few weeks to allow resolution of tributaries. That way minimalistic sclerotherapy or UGFS is required at follow up for cosmesis only.

Finally, recognition of an ischaemic attack can be difficult but prompt action for a suspected intra-arterial injection maybe the difference when it comes to saving the limb. Practitioners should develop a protocol for "suspected" arterial injection. However, no prospective human studies have shown that any specific treatment is superior [14] and the only universally recommended therapy is heparinisation, while steroids, acetyl-salicylic acid and peripheral vasodilators may all have their place.

\section{Conclusion}

It seems for now that sclerotherapy and UGFS will continue to be a mainstay of modern non-surgical varicose vein treatment. However, infrequent as tissue necrosis may be, it is truly devastating to both patient and physician when it occurs. Minimising the risk of complications by good patient selection, cautious treatment and developing adequate emergency protocols is vital and should not be forgotten.

Unfortunately complying with relevant guidelines can be difficult due to the heterogeneity of recommendations. In the future communication and collaboration between interested colleges and societies should be encouraged to improve the quality of data and development of more authoritative guidelines for sclerotherapy treatment.

\section{Conflicts of Interest}

No Conflicts of Interest.

\section{Funding}

No funding received for this publication.

\section{References}

1. Gloviczki P, Comerota AJ, Dalsing MC, Eklof BG, Gillespie $\mathrm{DL}$, et al. (2011) The care of patients with varicose veins and associated chronic venous diseases: Clinical practice guidelines of the society for vascular surgery and the american venous forum. J Vasc Surg 53: 2S-48S.

2. Gianesini S, Obi A, Onida S, Baccellieri D, Bissacco D, et al. (2019) Global guidelines trends and controversies in lower limb venous and lymphatic disease: Narrative literature revision and experts' opinions following the vWINter international meeting in Phlebology, Lymphology \& Aesthetics, 23-25 January 2019. Phlebology 34: 4-66. 
3. Yiannakopoulou E (2016) Safety concerns for sclerotherapy of telangiectases, reticular and varicose veins. Pharmacology 98: 62-69.

4. Hafner F, Froehlich H, Gary T, Brodmann M (2013) Intra-arterial injection, a rare but serious complication of sclerotherapy. Phlebology 28: 64-73.

5. Mwipatayi BP, Western CE, Wong J, Angel D (2016) Atypical leg ulcers after sclerotherapy for treatment of varicose veins: Case reports and literature review. Int J Surg Case Rep 25: 161-164.

6. Callum K, Bradbury A (2000) ABC of arterial and venous disease Acute limb ischaemia. BMJ 320: 764-767.

7. Bergan JJ, Weiss RA, Goldman MP (2000) Extensive tissue necrosis following high-Concentration sclerotherapy for varicose veins. Dermatol Surg 26: 535-541.

8. Tran D, Parsi K (2007) Veno-arteriolar reflex vasospasm of small saphenous artery complicating sclerotherapy of the small saphenous vein. Australian \& New Zealand Journal of Phlebology 10: 29-32.

9. Silva H, Ferreira HA, da Silva HP, Monteiro Rodrigues L
(2018) The venoarteriolar reflex significantlyreduces contralateral perfusion as part of the lower limb circulatory homeostasis in vivo. Front Physiol 9: 1123.

10. Ubbink DT, Jacobs MJ, Slaaf DW, Tangelder GJ, Reneman RS (1992) Microvascular reactivity differences between the two legs of patients with unilateral lower limb ischaemia. Eur J Vasc Surg 6: 269-275.

11. Uccioli L, Meloni M, Izzo V, Giurato L, Merolla S, et al. (2018) Critical limb ischemia: Current challenges andfuture prospects. Vasc Health Risk Manag 14: 63-74.

12. Sibley RC 3rd, Reis SP, MacFarlane JJ, Reddick MA, Kalva SP, et al. (2017) Noninvasive physiologic vascular studies: A guide to diagnosing peripheral arterial disease. Radiographics 37: 346-357.

13. Hwang JY (2017) Doppler ultrasonography of the lower extremity arteries: Anatomy and scanning guidelines. Ultrasonography 36: 111-119.

14. Sen S, Chini EN, Brown MJ (2005) Complications after unintentional intra-arterial injection of drugs: risks, outcomes, and management strategies. Mayo Clin Proc 80: 783-795. 